\title{
Nonlinear Viscoelasticity of Fiber-Filled Liquid Crystaline Polymer Melts
}

\author{
ARAKI Katsuhiko ${ }^{*}$ KITANO Takeshi ${ }^{* *}$ and MAEKAWA Zen-ichiro ${ }^{* * *}$ \\ *Industrial Technology Center of Fukui Prefecture, 6-12 \\ Ozowara, Miyazaki-Mura, Nyu-gun, Fukui, 916-0273 Japan \\ ${ }^{* *}$ National Institute of Advanced Industrial Science and Technology \\ 1-1-1 Higashi, Tsukuba, Ibaragi, 305-8561 Japan \\ ${ }^{* * *}$ Graduated School of Kyoto Institute of Technology, Matsugasaki \\ Sakyo-ku, Kyoto, 606-8585 Japan \\ Based on Textile Machinery Society of Japan, vol.54,No.8,T126-T134(2000-8)
}

\begin{abstract}
Dynamic viscoelasticity was measured for melts of semi-aromatic liquid crystalline polymer ( LCP ) filled with carbon fiber ( CF ) and glass fiber ( GF ) to quantitatively analyze their nonlinear viscoelastic properties. LCP and its CF and GF filled polymer systems were examined to analyze the dynamic viscoelastic behaviors by Fourier expansion of shear stress wave. For LCP the tertiary terms $\mathrm{G}_{3}$ and $\mathrm{G}_{3}$ are considerably smaller than their primary terms $\mathrm{G}_{1}{ }_{1}$ and $\mathrm{G}_{1},{ }_{1}$; the viscoelastic property is almost linear. On the other hand, two types of CF filled LCP showed that an increase in the oscillatory angle decreased $\mathrm{G}^{\prime}{ }_{i}$ and $\mathrm{G}{ }_{i}$ in sequence with increasing i. However, the $\mathrm{G}_{3}{ }_{3}$ and $\mathrm{G}{ }_{3}$ values are large enough not to be negligible, i.e. both fiber filled systems show similar nonlinear viscoelastic behaviors. GF filled LCP showed the tendency similar to that for CF filled systems; both $\mathrm{G}^{\prime}{ }_{i}$ and $G{ }^{\prime}{ }_{i}$ have apparently a plateau region. Furthermore, the values of the ratios $G_{3} / G_{1}, G^{\prime}{ }_{3} / G^{\prime}{ }_{1}$ and $\mathrm{G}{ }_{3} / \mathrm{G}{ }_{1}$ are recognized as the indicator of the degrees of their nonlinearity, and the fiber filled LCP systems showed no significant difference in their dependency on $\omega$, although GF filled systems showed a markedly higher nonlinearity of $G{ }_{3} / G{ }^{\prime}{ }_{1}$ than $\mathrm{CF}$ filled systems in a high $\omega$ region.
\end{abstract}

Keywords : Nonlinear viscoelastic property, liquid crystalline polymer, fiber filled polymer melts, storage and loss modulus, Lissajous figure

\section{Introduction}

It is well known that liquid crystalline polymers show unique rheological properties different from those of polyethylene, polystyrene, nylon and other general-purpose polymers [1-11]. Especially in their molten state, the liquid crystalline polymers demonstrate characteristic features such as marked shear rate dependency and low viscosity compared to those of such conventional polymers. In addition, a liquid crystalline polymer is characterized by its main chain so rigid as to allow it to be molten with little bending of its molecular chains, which can be highly oriented when it is subjected to slight shearing, making it crystalline while in a liquid state [12]. These characteristic properties of liquid crystalline polymers allow them to be categorized as one of highly functional materials for use in mechanical and electronic component parts, as well as in other special coating fields where they are molten and molded for use.

In recent years, various types of liquid crystalline polymers with different chemical structures have been made available on a commercial basis, while fully aromatic liquid crystalline polymers such as Vectra (VA) and their composites have been investigated by the authors and others to clarify their viscoelastic properties for the establishment of their optimum molding conditions. The authors have conducted the researches [1-5], [8-10] on the melts of fully aromatic liquid crystalline polymer VA and semi-aromatic liquid crystalline polymer Novaculate (NE), as well as their glass fiber (GF), carbon fiber (CF) and carbon black (CB) filled systems to investigate the flow properties of the melts. From a series of experimental researches on $\mathrm{CF}$, GF, and $\mathrm{CB}$ filled systems, the following results were obtained. The steady-state shear viscosity $\eta$ is dependent on their filler volume fraction $\left(\phi_{v}\right)$ in the same manner as with an ordinary chain polymer, in which the dependency of $\eta$ on

*Corresponding author Fax:0778-32-2005, E-mail: araki@vcnet.fukui.fukui.jp 
$\phi_{v}$ decreases with increasing temperature. The VA systems shows a markedly non-Newtonian flow, while the NE systems shows a Newtonian flow. Both the VA and NE systems show large dependency of storage modulus $G$ ', loss modulus G" and dynamic viscosity $\eta$ ' on angular frequency $\omega$ and filler volume fraction $\phi_{v}$, in which the dependency of G', G', and dynamic viscosity $\eta$ ' on $\omega$ is larger in their $\mathrm{CB}$ filled systems than in their fiber filled systems. VA systems show a dilatant flow behavior, which disappears in their $\mathrm{GF} / \mathrm{CF}$ and $\mathrm{CB}$ filled systems with an increase in the filler content or a rise in the temperature. As described above, VA, NE and CB filled systems showed flow properties peculiar to composite liquid crystalline polymers. In addition, the authors have further researched on these VA and NE liquid crystalline polymer composites to identify the effects of their molding conditions and oscillation modes (stretching/bending) on the dependency of their solid viscoelastic properties - storage modulus E', loss modulus E" and loss tangent $\tan \delta$ - upon their fiber contents and angular frequency $\omega$ as reported [6], [7].

When tested for their viscoelasticity in molten state, short fiber or particle filled composite polymer melts or polymer suspensions often give Lissajous's figures in shape not ellipse, but skewed. This is because the polymer melt or suspension system assumes to show a nonlinear viscoelastic behavior, even if flowed relatively under small strain. This suggests that it is important to take into consideration such non-linearity of the polymer system for exact analysis of its dynamic viscoelasticity. Notwithstanding this, however, there is no established method available to analyze such non-linear viscoelasticity, although there are some reports on a method for such analysis and the experimental results [1317]. Based on the Fourier expansion analysis of stress curve under sinusoidal strain, non-linear viscoelastic functions, $G_{i}{ }_{i}$ and $G{ }_{i}$ are determined. Such correction of the functions with addition of higher order frequency terms has been reported to allow the stress curve based analysis to be made with relatively good reproducibility [13-16].

While having researched fiber filled liquid crystalline polymers and other similar composite polymer systems, the authors have often observed their melts to show non-linear viscoelastic behavior. Notwithstanding this, the authors have thus far made reports on the research, ignoring such behavior of the polymer melts to treat them as linear viscoelastic [1], [9]. In the present research, the authors conducted experiments on the melts of semi-aromatic liquid crystalline polymers and the systems filled with short carbon and glass fibers by measuring their dynamic viscoelasticity using a cone-plate type viscometer for analysis of their nonlinearity by Fourier transform [13-17] to make one attempt to revise the relevant research results presented by the authors in the past.

\section{Experimental}

\subsection{Materials}

The specimens used were prepared from a polyester copolymer of p-hydroxy benzoic acid (PHB) and polyethylene terephthalate (PET) obtained as a liquid crystalline polymer (LCP, L5000, RODRUN, UNITIKA), carbon fiber (CF, diameter "d" $=7 \mu \mathrm{m}$ ) filled LCP (CF/LCP-1, LC5020) and glass fiber (GF, $d=11 \mu \mathrm{m})$ filled LCP (GF/LCP, LG5030), all of which are commercially available as pellets from UNITIKA. In addition, LCP and CF (BESFIGHT HTA-12000f, TOHO RAYON) were compounded by the same procedure as described in the authors' previous paper [1], and extruded into pellets for use as specimens (CF/LCP-2). For their rheological measurements, these four types of pellets were molded under compression at the temperature of $280^{\circ} \mathrm{C}$ into $3-\mathrm{mm}$ thick plates and then cut into discs of approximately $30 \mathrm{~mm}$ in diameter. The contents of CF or GF in the specimens (represented as its weight fraction $\phi_{\mathrm{w}}$ and volume fraction $\left.\phi_{v}\right)$ and the ratio of its length " $l_{m}$ " to its diameter " $d$ " (represented as its aspect ratio $\mathrm{a}_{\mathrm{r}}$ ) were also measured using the same methods as described in the previous paper [1]. The results of the measurements are shown in Table 1.

Table 1 Sample code, fiber content, size and aspect ratio of fiber dispersed in liquid crystalline polymers at various oscillatory angles.

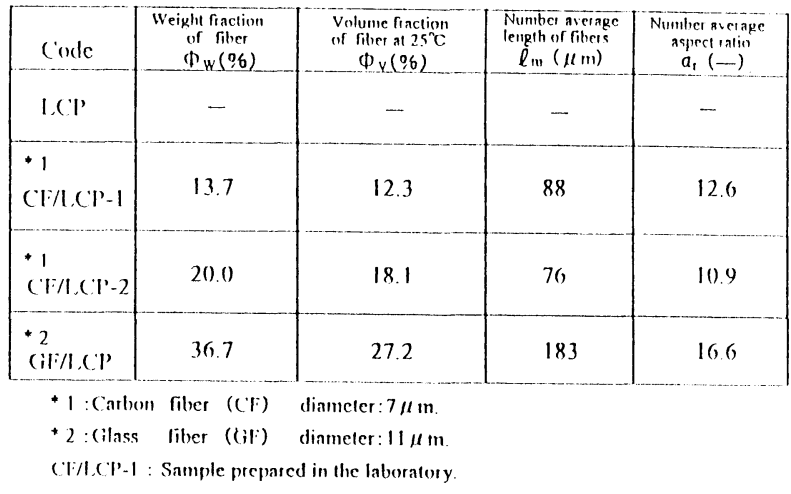

\subsection{Rheological Measurements}

The measurements of the steady-state flow properties in the low shear rate region were conducted using a cone-plate type rheogoniometer (RGM152-SD, NIPPON RHEOLOGY KIKI) with a cone radius " $R$ " of $2.15 \mathrm{~cm}$, a cone-plate gap " $h$ " of $175 \mu \mathrm{m}$ and a cone angle " $\mathrm{d}$ " of $4^{\circ}$ under a nitrogen atmosphere at a temperature of $280^{\circ} \mathrm{C}$. The shear rate $\gamma$ and viscosity $\eta$ in steady shear flow were determined according to the methods described in the previous paper [1]. 
In addition, the dynamic viscoelastic properties by storage modulus G' and loss modulus G" were measured using the above apparatus in the angular frequency " $\omega$ " range of 0.001 to $20(1 / \mathrm{s})$ at different oscillatory angles " $\theta$ " of $0.5^{\circ}$, $2^{\circ}$ and $5^{\circ}$, which correspond to strain amplitudes " $\gamma$ " of $0.125,0.5$ and 1.25 , respectively. All the measurements were carried at the temperature of $280^{\circ} \mathrm{C}$.

The results of the measurements showed that the specimens gave two types of stress curves in response to their sinusoidal strain - one is sinusoidal, indicating that they are of linear viscoclasticity and the other is non-sinusoidal, indicating that they are of non-linear viscoelasticity.

On the other hand, the specimens gave two types of Lissajous's figures obtained by X-Y recorder - one is of ellipse, indicating that they are of linear viscoelasticity, and the other is of skewed figures, indicating that they are of non-linear viscoelasticity, the latter of which was more frequently observed in the present research. The method for analyzing the non-linear viscoelasticity data is shown in the following section.

\subsection{Method for Analysis of Non-linear Viscoelasticity}

The rheological properties of the specimens were measured using a cone-plate type rheogoniometer with sinusoidal oscillation applied to the cone or plate. The stress waves transmitted to the cone or plate were quantitatively analysed by Fourier expansion to determine their non-linear complex elastic modulus components $\mathrm{G}^{\prime}{ }_{\mathrm{i}}$ and $\mathrm{G}{ }_{\mathrm{i}}$. The coneplate type rheogoniometer used in the present experiments specifies the use of the following equation in determining the viscoelasticity G' and G" of the specimens if it is linear:

$$
\mathrm{G}^{\prime}=\omega \eta \eta^{\prime}, \mathrm{G}^{\prime}=\omega \eta^{\prime}
$$

where $\omega$ indicates the angular frequency, $\omega \eta "=\left(\mathrm{A}_{1} \mathrm{M}_{0} / \omega\right.$ $\left.\theta^{\circ}\right) \cos \delta$ and $\eta^{\prime}=\left(\mathrm{A}_{1} \mathrm{M}_{0} / \omega \theta\right) \sin \delta$, in which $\mathrm{A}_{1}$ represents the geometrical constant given as $\mathrm{A}_{1}=3 \alpha / 2 \pi \mathrm{R}^{3}$, $\mathrm{M}_{0}$ the torque amplitude, $\delta$ the phase angle, $\alpha$ the cone angle, $\theta$ the oscillatory angle and $\mathrm{R}$ the cone radius.

The Lissajous's figure obtained for the particular specimen from the dynamic viscoelasticity measurement understandably indicates whether its viscoelasticity is non-linear according the extent of distortion of the figure, though only on a qualitative basis. To determine the non-linear viscoelasticity of the particular specimen quantitatively, the stress curve obtained was subjected to numerical Fourier transformation to examine for introduction of correction terms - 1st-, 2nd- and 3rd-order. The shear stress including the higher harmonic wave components is expressed by the following equation:

$$
\tau=\sum\left(G^{\prime}{ }_{i} \gamma_{0}{ }^{i} \sin i \omega t+G{ }^{\prime}{ }_{i} \gamma_{0}{ }^{i} \cos i \omega t\right)
$$

where $G_{i}$ and $G{ }_{i}(i=1,2 \ldots)$ indicate the dynamic clastic moduli of the specimen when its viscoelasticity is nonlinear; $G{ }_{1}$ and $G{ }_{1}$, respectively, correspond to $G$ ' and $G$ " expressed by formulas (1) for linear viscoelasticity, representing the elastic storage energy $\left(\mathrm{G}^{\prime}{ }_{1}\right)$ and thermallydissipating energy $\left(\mathrm{G}_{1}\right)$ input into or output from the system during one cycle. The oscillatory (shear) strain is expressed by the following equation:

$$
\gamma=\gamma_{0} \sin \omega t
$$

In Equation 2 , the even terms, for example, $\left(\mathrm{G}_{2}{ }_{2} \gamma_{0}{ }^{2} \sin 2 \omega\right.$ $\left.\mathrm{t}+\mathrm{G}{ }_{2} \gamma_{0}{ }_{0}^{2} \cos 2 \omega \mathrm{t}\right)$ are even functions showing positive and negative components symmetrical to each other against the " $\mathrm{t}$ " axis (horizontal) during one cycle, causing $\mathrm{G}_{2}$, and $\mathrm{G}_{2}, \mathrm{G}_{4}$ and $\mathrm{G}{ }_{4}$, and other even terms to become zero, therefore the following equation is induced:

$\tau=\mathrm{G}_{1}{ }_{1} \gamma_{0}{ }_{0}^{1} \sin 1 \omega \mathrm{t}+\mathrm{G}{ }^{\prime}{ }_{1} \gamma_{0}{ }^{1} \cos 1 \omega \mathrm{t}-\mathrm{G}_{3}{ }_{3} \gamma_{0}{ }_{0}^{3} \sin$ $3 \omega \mathrm{t}-\mathrm{G}{ }_{3} \gamma_{0}{ }_{0}^{3} \cos 3 \omega \mathrm{t}+\mathrm{G}_{5}{ }_{5} \gamma_{0}^{5} \sin 5 \omega \mathrm{t}+\mathrm{G}{ }_{5}{ }_{5} \gamma_{0}{ }^{5} \cos 5$ $\omega \mathrm{t}-\cdots$

$\mathrm{G}^{\prime}{ }_{1}$ and $\mathrm{G}{ }_{1}$, values are used for analysis without addition of their higher terms $\mathrm{G}_{3} / \mathrm{G}_{3}$ and $\mathrm{G}{ }_{5} / \mathrm{G}{ }_{5}$ if the values of these terms are negligibly small (being approximately $1 / 10$ or below), but require correction with addition of either or both of these higher terms if their values are large, so as to allow approximation to the measured non-linear response curve.

\section{Results and Discussions}

\subsection{Steady shear flow properties of LCP and its fiber- filled systems}

For the semi-aromatic liquid crystalline polymer LCP, at $280^{\circ} \mathrm{C}$ relations between steady-shear flow viscosity $\eta$ and shear rate $\gamma$ are shown in Fig. 1 together with those for its carbon fiber (CF) and glass fiber (GF) filled systems. The unfilled LCP shows a gradual linear decrease in $\eta$ with an increase of $\gamma$. On the other hands, all the fiber filled LCP give $\eta-\gamma$ curves showing remarkable non-Newtonian behavior compared to their unfilled counterpart. The unfilled LCP shows a low viscosity of approximately $10^{2}$ $\mathrm{Pa} \cdot \mathrm{s}$ at the shear rate of $10^{-1}\left(\mathrm{~s}^{-1}\right)$. In contrast, the viscosity of the fiber-filled LCP in the low $\gamma$ region is larger than that of their unfilled LCP counterpart by more than two decade. It should be noted here that the CF/LCP-1 specimen with a fiber volume fraction $\phi_{\mathrm{v}}$ of $12.3 \%$ gave a larger viscosity value than its other $\mathrm{CF}$ filled system, CF/LCP-2 whose $\phi_{v}$ is $18.1 \%$. Generally, the viscosity of a fiber-filled polymer system tends to increase with an increase of fiber content. However, the results obtained 


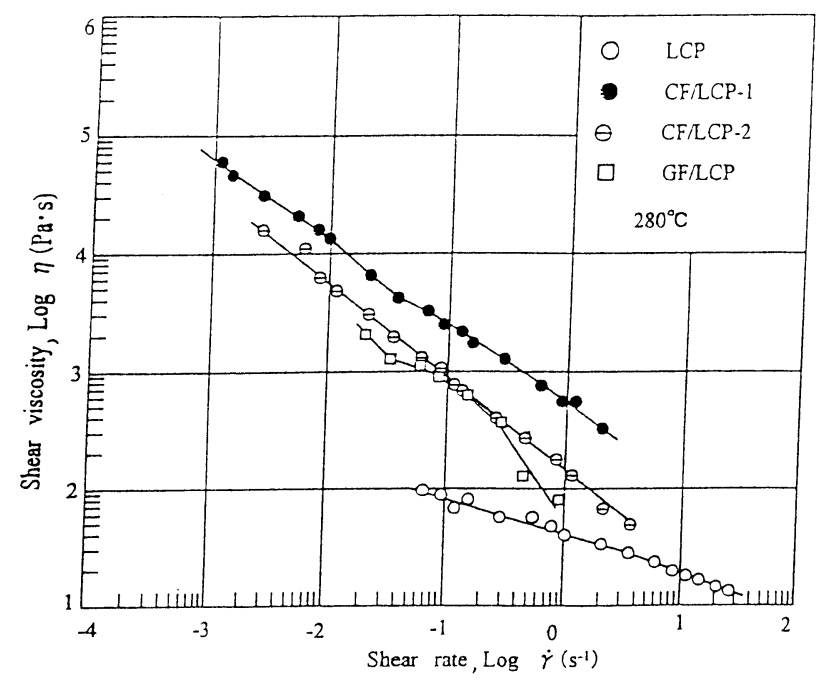

Fig.1 Relationship between shear viscosity and shear rate of LCP and fiber filled LCP systems at $280^{\circ} \mathrm{C}$.

from the CF/LCP-1 and CF/LCP-2 are to the contrary as shown in Table 1, suggesting that they are attributable to the difference in the length of the fiber. It also should be noted that the CF/LCP-1 was prepared using an elastic melt extruder, allowing its $\mathrm{CF}$ to undergo less damage than that of the commercially obtained CF/LCP-2. In addition, the $\eta-\gamma$ curves of the fiber-filled LCP suggest the existence of yield stress. The analysis method for yield stress and the results of the experiments have been reported in the previous papers [1] [9].

\subsection{Viscoelastic properties of unfilled LCP}

Fig. 2 shows the storage modulus $G_{i}(i=1,3)$ and loss modulus $\mathrm{G}{ }_{i}(\mathrm{i}=1,3)$ of the unfilled LCP melt measured at $280^{\circ} \mathrm{C}$ as a function of angular frequency $\omega$ at the oscillatory angles $\theta$ (strain amplitude $\gamma$ ) of $2^{\circ} \quad(\gamma=0.5)$ and $5^{\circ}(\gamma=1.25)$. The LCP melt gave a Lissajous's figure of unskewt shape (though not shown). In addition, the LCP melt showed no marked non-Newtonian and non-linear viscoelastic behavior compared to that of a fully-aromatic liquid crystalline polymer such as Vectra, while it presented no dilatant flow property. $G{ }_{1}(\triangle)$ and $G{ }^{\prime}{ }_{1}(\bigcirc)$ at the oscillatory angle of $2^{\circ}$ increase almost in proportion to angular frequency $\omega$ in the relatively low $\omega$ region of $10^{-1}<\omega<10\left(\mathrm{~s}^{-1}\right)$. The $\mathrm{G}^{\prime}{ }_{1}$ and $\mathrm{G}^{\prime}{ }_{1}$ curves obtained at the larger oscillatory angle of $5^{\circ}$ almost overlap their respective values at the oscillatory angle of $2^{\circ}$, suggesting that the influence of the oscillatory angle or strain amplitude on the viscoelasticity is small.

In addition, it is found from Fig. 2 that the 3 rdorder terms of G' and G" (representing the non-linearity of the specimen's viscoelasticity) are smaller than their

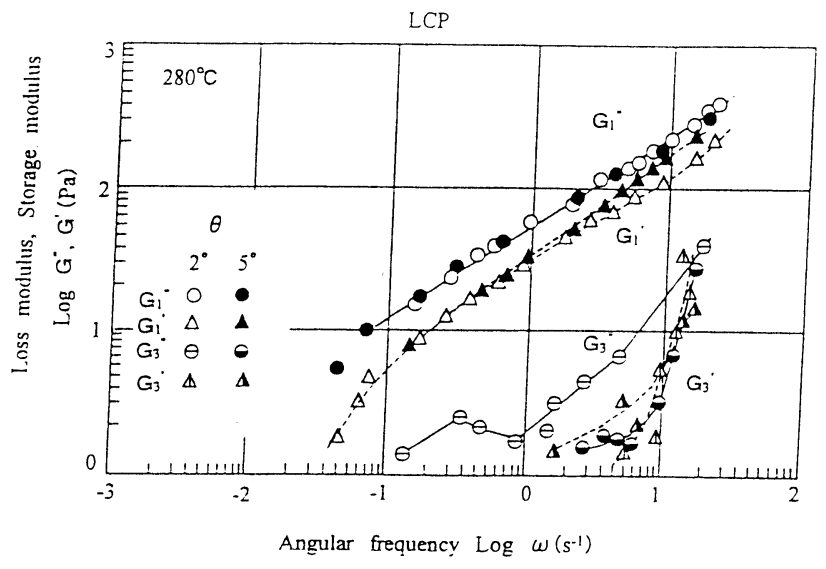

Fig. 2 Loss modulus $G{ }^{\prime}{ }_{i}$ and storage modulus $\mathrm{G}^{\prime}{ }_{i}$ of LCP as a function of angular frequency at the oscillatory angles $(\theta)$ of 2 and 5 degs.

respective 1 st-order terms $G{ }^{\prime}{ }_{1}$ and $G "{ }_{1}$ all over the $\omega$ region by more than one decade. Although not shown here, the higher order terms of G' and G' became further smaller. This suggests that of the unfilled LCP shows nearly linear viscoelasticity.

\subsection{Viscoelasticity of fiber-filled LCP}

\subsubsection{Non-linear viscoelasticity of CF/LCP}

Viscoelastic properties of CF/LCP-1 $\left(\phi_{\mathrm{v}}=12.3 \%\right)$ was measured at the oscillatory angles, $\theta$ of $0.5^{\circ}, 2^{\circ}$ and $5^{\circ}$ at $280^{\circ} \mathrm{C}$, and storage modulus $\mathrm{G}_{\mathrm{i}}^{\prime}(\mathrm{i}=1,3,5)$ and loss modulus $G{ }_{i}(i=1,3,5)$ as a function of angular frequency, $\omega$ were analysed. The results were shown in Fig. 3 and Fig.4. Based on these results, the 1st-order term of the Fourier series and their higher-order terms (3rd/5th) representing the extent of the non-linearity of the vicoelasticity are examined for their dependency on the angular frequency $\omega$ at three different oscillatory angles as described below. From the results obtained at $\theta=0.5^{\circ}$ (strain amplitude $\gamma=0.125$ ), $G^{\prime}{ }_{1}$ and $G^{\prime}{ }_{1}$ show largely different $\omega$ dependence in the range of $10^{-2}<\omega<10\left(\mathrm{~s}^{-1}\right)$ in comparison with the unfilled LCP (Fig.2); specifically, both $\mathrm{G}_{1}$ and $\mathrm{G}{ }_{1}$, have definitely plateau regions zones, showing small $\omega$ dependency. In addition, $\mathrm{G}_{3}$ and $\mathrm{G}_{3}$ are both smaller than their 1 st-order terms $\mathrm{G}^{\prime}{ }_{1}$ and $G{ }^{\prime}{ }_{1}$ all over the measured $\omega$ region, showing small $\omega$ dependency like that of the latter. Further, $\mathrm{G}_{5}{ }_{5}$ and $\mathrm{G}{ }_{5}$ are both still smaller than their 3rd-order counterparts all over the measured $\omega$ region, similarly showing small $\omega$ dependency, which tends to decrease in the high $\omega$ region. It should be noted that the values of the 3 rd-order terms $\mathrm{G}_{3}$ and $\mathrm{G}_{3}$ are of such magnitude as not to be negligible compared to those of their 1 st-order counterparts (1/10 or above), suggesting that 
$\mathrm{CF} / \mathrm{LCP}-1$
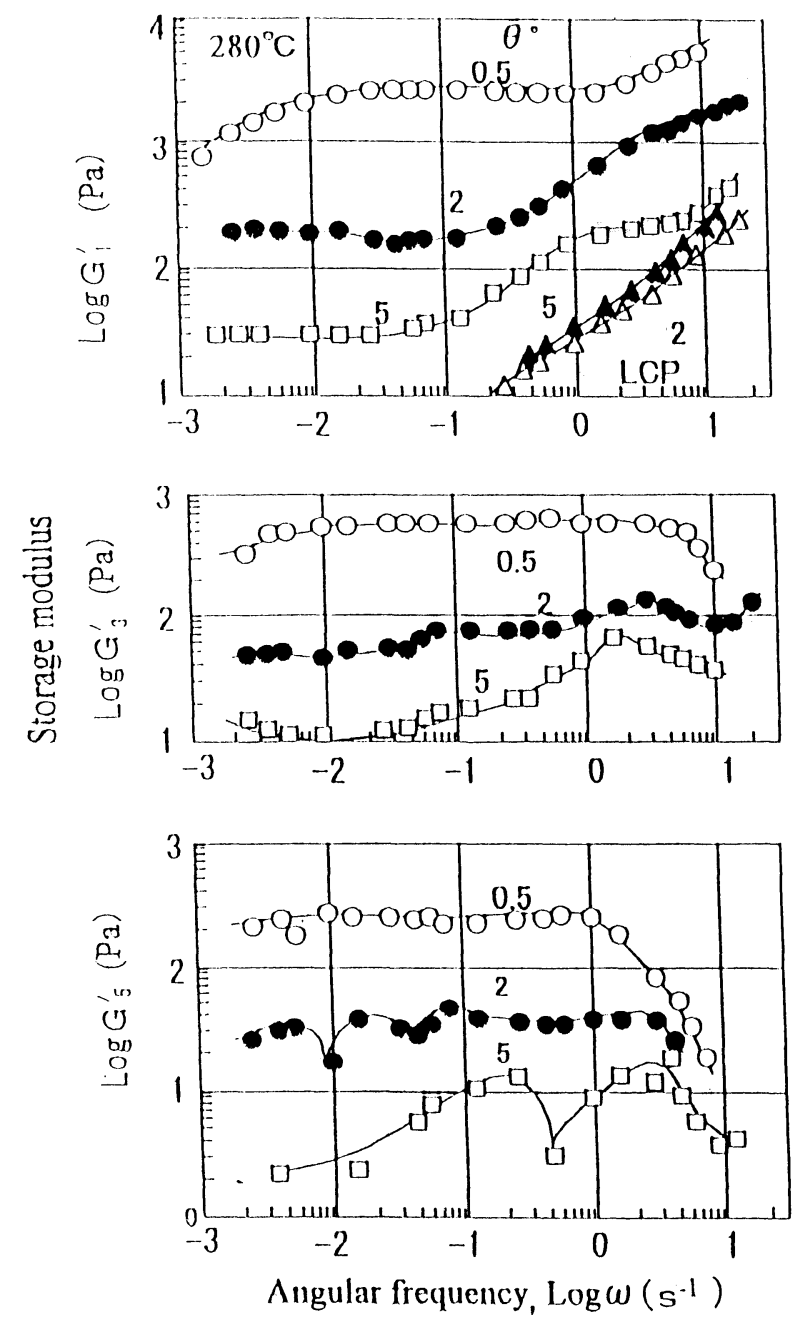

Fig. 3 Storage modulus ( $1^{\text {st }}$ order, $G{ }_{1} 3^{\text {rd }}$ order, $\mathrm{G}_{3}$ and $5^{\text {th }}$ order, $\mathrm{G}_{5}$ ) of CF/LCP-1 as a function of angular frequency at various oscillatory angles.

$\mathrm{CF} / \mathrm{LCP}$ represents the non-linear viscoelasticity.

From the results obtained at $\theta=2^{\circ}$ (strain amplitude $\gamma$ $=0.5$ ), it is found both $\mathrm{G}^{\prime}{ }_{1}$ and $\mathrm{G}{ }_{1}$ tend to be smaller than those at $\theta=0.5^{\circ}$ all over the $\omega$ range by approximately $1 / 10$. This indicates that both of them markedly decrease with a rise in the oscillatory angle, which fact, in turn, suggests that their dependency on the oscillatory angle is remarkable. In addition, the $\mathrm{G}^{\prime}{ }_{1}$ curve shows $\omega$ dependence in the $\omega>0.1\left(\mathrm{~s}^{-1}\right)$ range. With regard to $\mathrm{G}_{3}{ }_{3}$ and $\mathrm{G}_{3}$, both, similarly to those obtained at $\theta=0.5^{\circ}$, are smaller than $G^{\prime}{ }_{1}$ and $G^{\prime}{ }_{1}$, and their $\omega$ dependency is also small. In addition, $\mathrm{G}_{5}{ }_{5}$ and $\mathrm{G}{ }_{5}$ are markedly smaller than their respective 1st-order counterparts, also showing small $\omega$ dependency. This means that the correlation of these 3 rd- and 5 th-order term values with their 1 st-order counterparts is similar to that obtained at $\theta=0.5^{\circ}$. It should be noted that the values of the 3 rd-order terms $\mathrm{G}_{3}$
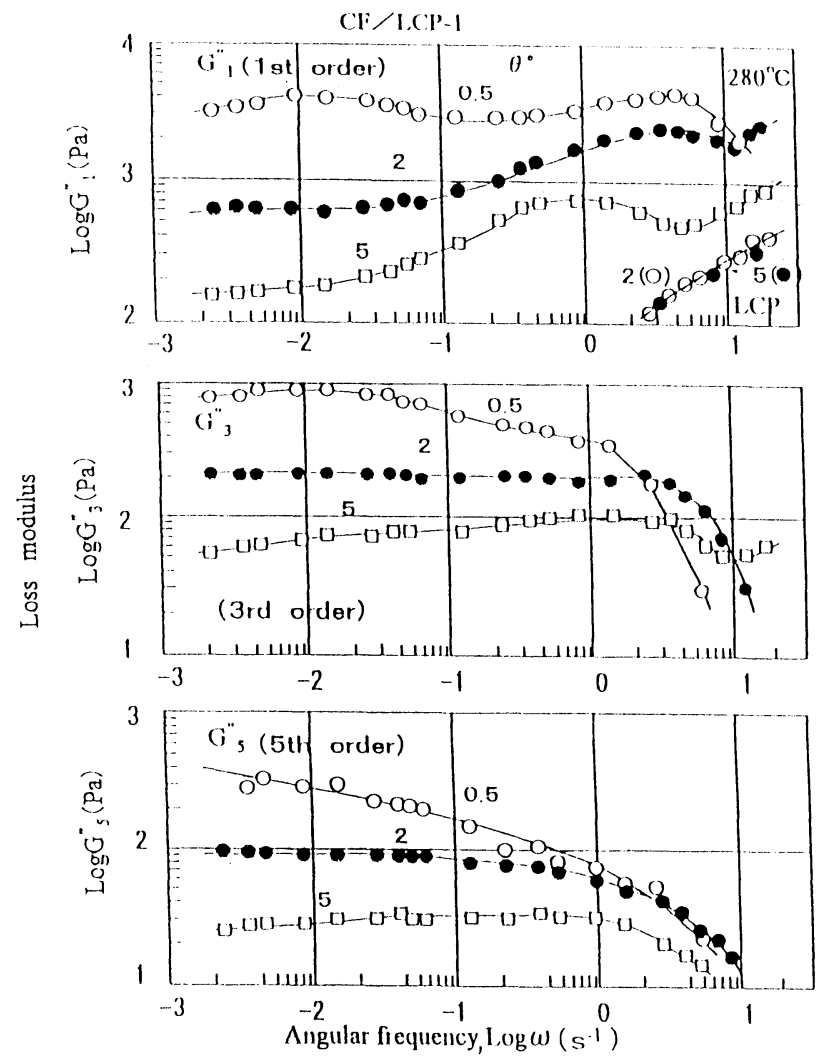

Fig.4 Loss modulus ( $\left({ }_{1},{ }_{1},{ }_{3}\right.$ and $G{ }_{5}$ ) of CF/LCP-1 as a function of angular frequency at various oscillatory angles.

and $\mathrm{G}_{3}$ in the $\omega<0.1\left(\mathrm{~s}^{-1}\right)$ region are of such magnitude as not to be negligible compared to those of their $1 \mathrm{st}$-order counterparts $(1 / 10$ or above). This indicates that they represent the non-linearity of the viscoelasticity of CF/LCP. The above results suggest that both of the oscillatory angle and angular frequency are the important factors affecting the non-linearity of the viscoelasticity of $\mathrm{CF} / \mathrm{LCP}$.

The results obtained at various oscillatory angles in Figs. 3 and 4 show that of $\omega$ all $G{ }_{i}$ and $G{ }^{\prime}{ }_{i}$ decrease with a rise in $\theta$ all over the measured $\omega$ region. In addition, the values of the $G^{\prime}{ }_{i}$ and $G{ }_{i}$ terms decrease as their orders increase. It should be noted that the values of the 3rd-order terms $\mathrm{G}_{3}$ and $\mathrm{G}_{3}{ }_{3}$ are not negligible compared to those of their 1st-order terms. However, the values of the 5th-order terms $\mathrm{G}_{5}$ and $\mathrm{G}{ }_{5}$ are negligible as they are smaller than their respective 1st-order counterparts by almost 1 decade. The correlation of the values of the 1st-order terms with those of their higher-order counterparts is described later.

When we compare these results with those of unfilled LCP in Fig.3, it is found that CF/LCP shows a large rate of decrease in $\mathrm{G}_{1}{ }_{1}$ with an increase in $\theta$. This clearly represents its dependency on the fiber content and oscillatory angle or strain amplitude. In addition, the $\mathrm{G}_{1}$ curve for CF/LCP at $\theta=5^{\circ}$ tends to become more like its 
counterpart for LCP at $\theta=2^{\circ} / 5^{\circ}$ in the higher $\omega$ region. Further, three $\mathrm{G}_{1}$, curves for $\mathrm{CF} / \mathrm{LCP}-1$ (respectively obtained at $\theta=0.5^{\circ}, 2^{\circ}$ and $5^{\circ}$ ) have a definite plateau region (where dependency of $\mathrm{G}^{\prime}{ }_{1}$ on $\omega$ is small), which shifts to the lower $\omega$ region with a rise in $\theta$. This plateau region is not observed in the corresponding curves of the unfilled LCP, which shows a nearly linear viscoelastic behavior.

In reference to the relationship between $\mathrm{G}{ }_{i}$ and $\omega$ of CF/LCP, for CF/LCP-1 ( $\phi_{v}=12.3 \%$ ), Fig.4 summarizes the dependency of $G{ }_{1}, G{ }_{3}$ and $G{ }_{5}$ on $\omega$. The $G{ }_{1}$ curve of LCP is also shown for the comparison with that of CF/LCP. $G{ }_{1}$ value is larger than that of the latter, which maximizes at the smallest oscillatory angle of $0.5^{\circ}$, showing dependency of $G{ }_{1}$ on the fiber content. When the higher-order $G{ }{ }_{i}$ terms of $\mathrm{CF} / \mathrm{LCP}$ are compared with those of its unfilled counterpart (which shows considerable variation in $\mathrm{G}_{3}$ at $\theta$ of $2^{\circ}$ and $5^{\circ}$ as observed in Fig.2), it is found that both $G{ }_{3}$ and $G{ }_{5}$ of the former show a certain dependency on the oscillatory angle. The dependency of $G{ }_{3}$ on $\omega$, compared to that of the other two terms $G{ }_{1}$ and $G{ }_{5}$, is markedly small in the lower $\omega$ region of $\omega<1\left(\mathrm{~s}^{-1}\right)$ at almost all three measured oscillatory angles of $0.5^{\circ}, 2^{\circ}$ and $5^{\circ}$. In addition, all of the three $\mathrm{G}^{\prime}{ }_{5}$ curves obtained at each oscillatory angle show a small dependency of $G{ }{ }_{5}$ on the strain amplitude in the high $\omega$ region and its values decrease with an increase in $\omega$. As described above, the G” ${ }_{3}$ and $G{ }_{5}$ of the CF/LCP-1, which represent the non-linearity of its viscoelasticity, show a tendency to decrease sharply in the high $\omega$ region.

The results for CF/LCP-2 $\left(\phi_{\mathrm{v}}=18.1 \%\right)$ are shown in Fig.5. The relationship between $\mathrm{G}_{i}{ }_{i}$ and $\mathrm{G}{ }_{i}(\mathrm{i}=1,3,5)$ and angular frequency $\omega$ at oscillatory angle $\theta$ of $0.5^{\circ}$ is plotted. The relationship between $\mathrm{G}^{\prime}{ }_{1}$ and $\mathrm{G}^{\prime}{ }_{1}$ and $\omega$ at $\theta=$ $0.5^{\circ}, 2^{\circ}$ and $5^{\circ}$ is given in Fig. 6 together with similar data on $\mathrm{G}^{\prime}{ }_{1}$ and $\mathrm{G}^{\prime}{ }_{1}$ of the unfilled LCP at $\theta=2^{\circ}$ and $5^{\circ}$. The comparison of Figs. 5 and 6 with Figs. 3 and 4, which show similar data on CF/LCP-1 at $\theta=0.5^{\circ}$, indicates that both are similar in the dependency of $\mathrm{G}^{\prime}{ }_{i}$ and $\mathrm{G}_{{ }_{i}}$ on $\omega$. The CF/LCP-2, whose filler volume fraction $\left(\phi_{\mathrm{v}}=18.1 \%\right)$ is larger than that of the CF/LCP-1 $\left(\phi_{\mathrm{v}}=12.31 \%\right)$, shows that the $\mathrm{G}^{\prime}{ }_{i}$ and $G{ }^{\prime}{ }_{i}(i=1,3,5)$ values of the former all tend to be smaller than those of the latter. As mentioned in 3.1, this is considered to be attributable to the fact that although the fiber content of CF/LCP-2 is higher than that of the CF/LCP-1, the fiber length of the former is shorter than that of the latter, and then the $\mathrm{G}^{\prime}{ }_{i}$ and $\mathrm{G}{ }_{i}$ values of the former decrease. In the previous paper [1], the authors have reported that the viscoelastic behavior of the filled LCP is complexly influenced by the fiber structure itself, the formation of the liquid crystalline polymer domain, the destruction of the domain, under flow, due to the existence of the fiber and other factors. The results obtained in this study for two types of CF/LCP as shown in Fig.5 and Figs.3
$\mathrm{Cl} / \mathrm{LCP}-2$
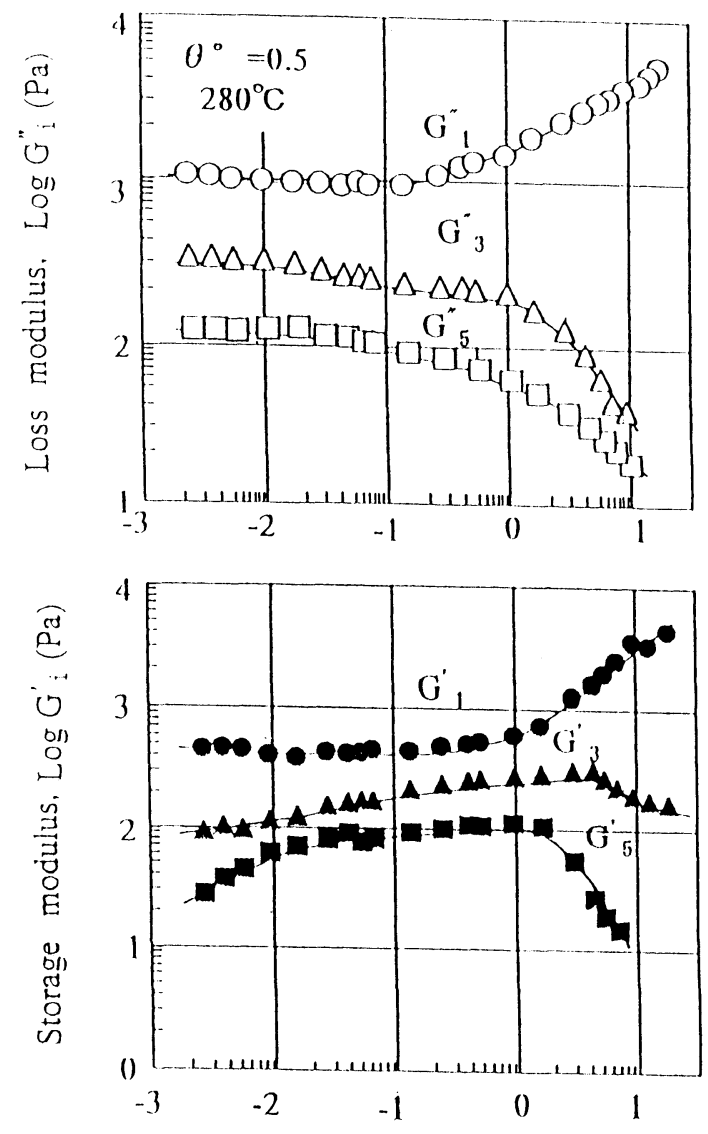

Angular frequency, $\log \omega\left(\mathrm{s}^{-1}\right)$

Fig.5 Loss modulus $\mathrm{G}^{\prime}{ }_{i}$ and storage modulus $\mathrm{G}_{i}$ of CF/LCP- 1 as a function of angular frequency $\left(\theta=0.5^{\circ}\right)$.

and 4, show that the non-linearity of the viscoelasticity of $\mathrm{CF} / \mathrm{LCP}$ is more influenced by fiber length than fiber content. These two types of CF/LCP are different from each other in their fiber content and fiber length distributions. $\mathrm{G}^{\prime}{ }_{i}$ and $\mathrm{G}{ }_{\mathrm{i}}(\mathrm{i}=1,3,5)$ in Fig.5 and Figs.3-4 are different in their values, but similar in their respective curves. When the results obtained for the CF/LCP-2 at $\phi=2^{\circ}$ and $5^{\circ}$ (not shown) are compared with those in Fig.4, it is found that they show similar $G_{i}$ and $G^{\prime}{ }_{i}$ curves. Their corresponding actual $\mathrm{G}_{i}$ and $\mathrm{G}{ }_{i}$ values, however, are considerably smaller than those for CF/LCP-1, as in the case at $\phi=0.5^{\circ}$.

With regard to the storage modulus $\mathrm{G}_{i}{ }_{i}$ of the CF/LCP-2, the dependency of its first-order term $\mathrm{G}^{\prime}{ }_{1}$ on the oscillatory angle $\theta$ is shown in Fig.6. Compared with Fig.3, the $\mathrm{G}_{1}{ }_{1}$ curve of the CF/LCP-2 at $\phi=0.5^{\circ}$ shows no dependency of $\mathrm{G}^{\prime}{ }_{1}$ on $\omega$ in the $\omega<1\left(\mathrm{~s}^{-1}\right)$ region, having a plateau region extending to the $\omega$ region, still lower than that of CF/LCP-1. However, the $\mathrm{G}_{1}{ }_{1}$ curves at $\phi=2^{\circ}$ and $5^{\circ}$ in Fig.6, which are largely different in shape from their counterparts in Fig.3, show large dependence of $\mathrm{G}_{1}{ }_{1}$ on $\omega$. 


\section{$\mathrm{CF} / \mathrm{L}, \mathrm{CP}-2$}
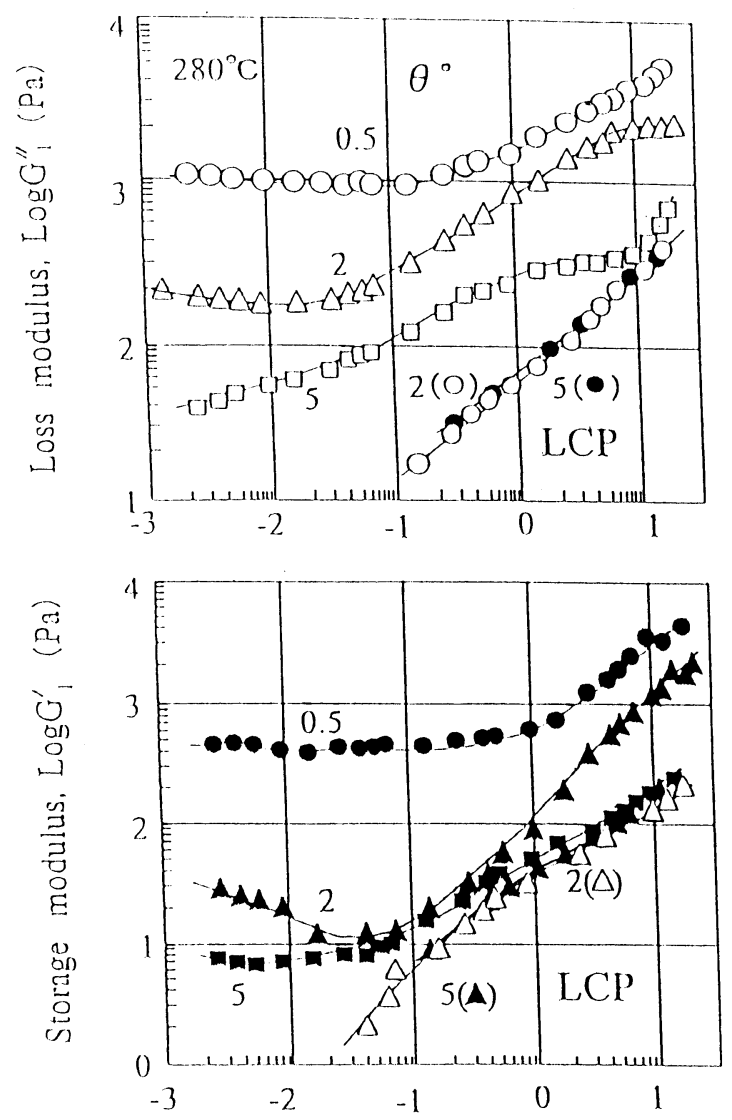

Angular frequency, $\log \omega\left(\mathrm{s}^{-1}\right)$

Fig. 6 Storage modulus ( $1^{\text {st }}$ order $) \mathrm{G}^{\prime}{ }_{1}$ and Loss modulus $\left(1^{\text {st }}\right.$ order) $\mathrm{G}{ }_{1}$ of $\mathrm{CF} / \mathrm{LCP}-1$ as a function of angular frequency at various oscillatory angles.

In addition, Fig. 6 shows that the $\mathrm{G}^{\prime}{ }_{1}$ curve at $\phi=5^{\circ}$ is more like that of the unfilled LCP plotted in the same figure with both overlapping in the $\omega \fallingdotseq 10\left(\mathrm{~s}^{-1}\right)$ region, similarly to the $\mathrm{G}_{1}{ }_{1}$ curve of CF/LCP-1 at $\phi=5^{\circ}$ as shown in Fig. 3, although the values of $G^{\prime}{ }_{1}$ in the $\omega<1\left(\mathrm{~s}^{-1}\right)$ region are smaller than those of $\mathrm{G}^{\prime}{ }_{1}$ given in Fig.3. This difference is considered to be largely due to the length of the fiber contained in CF/LCP-1 being larger than that in the CF/LCP-2, resulting in its higher-level structure formation.

Fig.6 also shows the dependency of the 1st-order term, $\mathrm{G}{ }_{1}$ of the loss modulus $\mathrm{G}{ }_{i}$ of CF/LCP-2 on the oscillatory angle $\theta$. Compared to the $G{ }_{1}$ values of $\mathrm{CF} / \mathrm{LCP}-1$ as shown in Fig.4, those of CF/LCP-2 in Fig.6 are small. However, three $\mathrm{G}_{1}$ curves in Fig.4 are characteristically similar in shape to those in Fig.6. The higher-order terms, $\mathrm{G}_{3}$ and $\mathrm{G}{ }_{5}$ of $\mathrm{G}{ }_{\mathrm{i}}$ of $\mathrm{CF} / \mathrm{LCP}-2$ (not shown) are both smaller than their respective counterparts of $\mathrm{CF} / \mathrm{LCP}-1$. This behavior also suggests that $G^{\prime}{ }_{i}$ of the fiber-filled LCP is more influenced by fiber length than fiber content.

\subsubsection{Non-linear Viscoelasticity of GF/LCP}

For GF/LCP $\left(\phi_{v}=27.2 \%\right)$, which is larger than CF/LCP both in the fiber content and fiber length (or aspect ratio), the relationship between $\mathrm{G}^{\prime}{ }_{i}$ and $\mathrm{G}^{\prime}{ }_{i}(\mathrm{i}=1,3,5)$ and $\omega$ at oscillatory angles $\theta$ of $0.5^{\circ}, 2^{\circ}$ and $5^{\circ}$ and at $280^{\circ} \mathrm{C}$ is shown in Fig.7 and Fig.8. Both $\mathrm{G}^{\prime}{ }_{\mathrm{i}}$ and $\mathrm{G}^{{ }_{\mathrm{i}}}$ at $\theta=0.5^{\circ}$ show a tendency to decrease as their respective orders increase, similarly to the case of CF/LCP. However, the comparison of $\mathrm{G}^{\prime}{ }_{i}$ and $\mathrm{G}{ }_{i}(\mathrm{i}=1,3,5)$ curves of GF/LCP with those of CF/LCP shows that the higher-order terms $\mathrm{G}^{\prime}{ }_{i}$ and $\mathrm{G}{ }_{\mathrm{i}}$ of $\mathrm{GF} / \mathrm{LCP}$ tend to become larger than those of $\mathrm{CF} / \mathrm{LCP}$. Especially, $\mathrm{G}_{3}$ and $\mathrm{G}_{3}{ }_{3}$ are large, suggesting that the non-linearity of the viscoelasticity of GF/LCP is larger than that of the two types of CF/LCP. In addition, $G^{\prime}{ }_{i}$ and

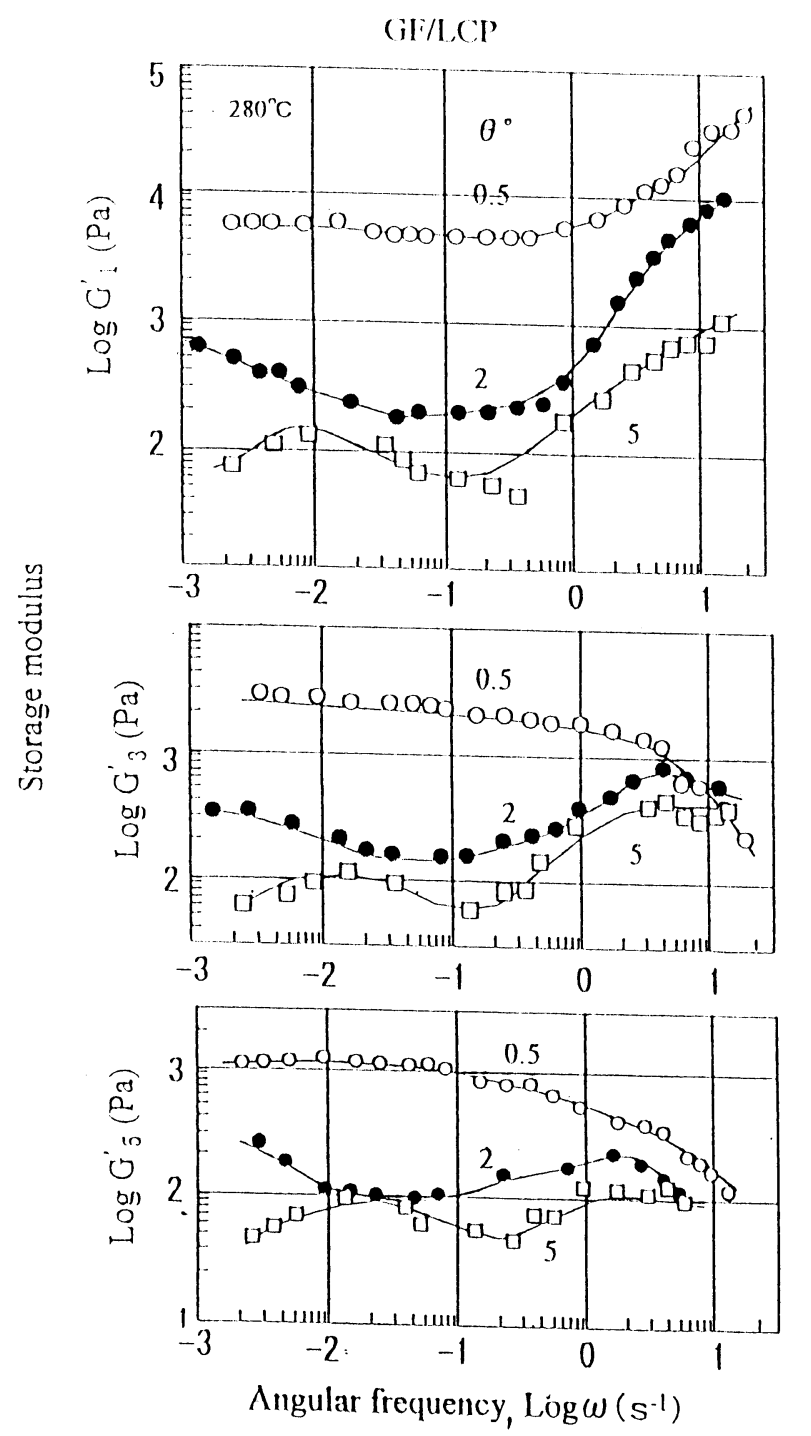

Fig.7 Storage modulus ( $1^{\text {st }}$ order, $\mathrm{G}_{1}{ }_{1} 3^{\text {rd }}$ order, $\mathrm{G}_{3}{ }_{3}$ and $5^{\text {th }}$ order, $\mathrm{G}_{5}{ }_{5}$ ) of $\mathrm{GF} / \mathrm{LCP}$ as a function of angular frequency at various oscillatory angles. 


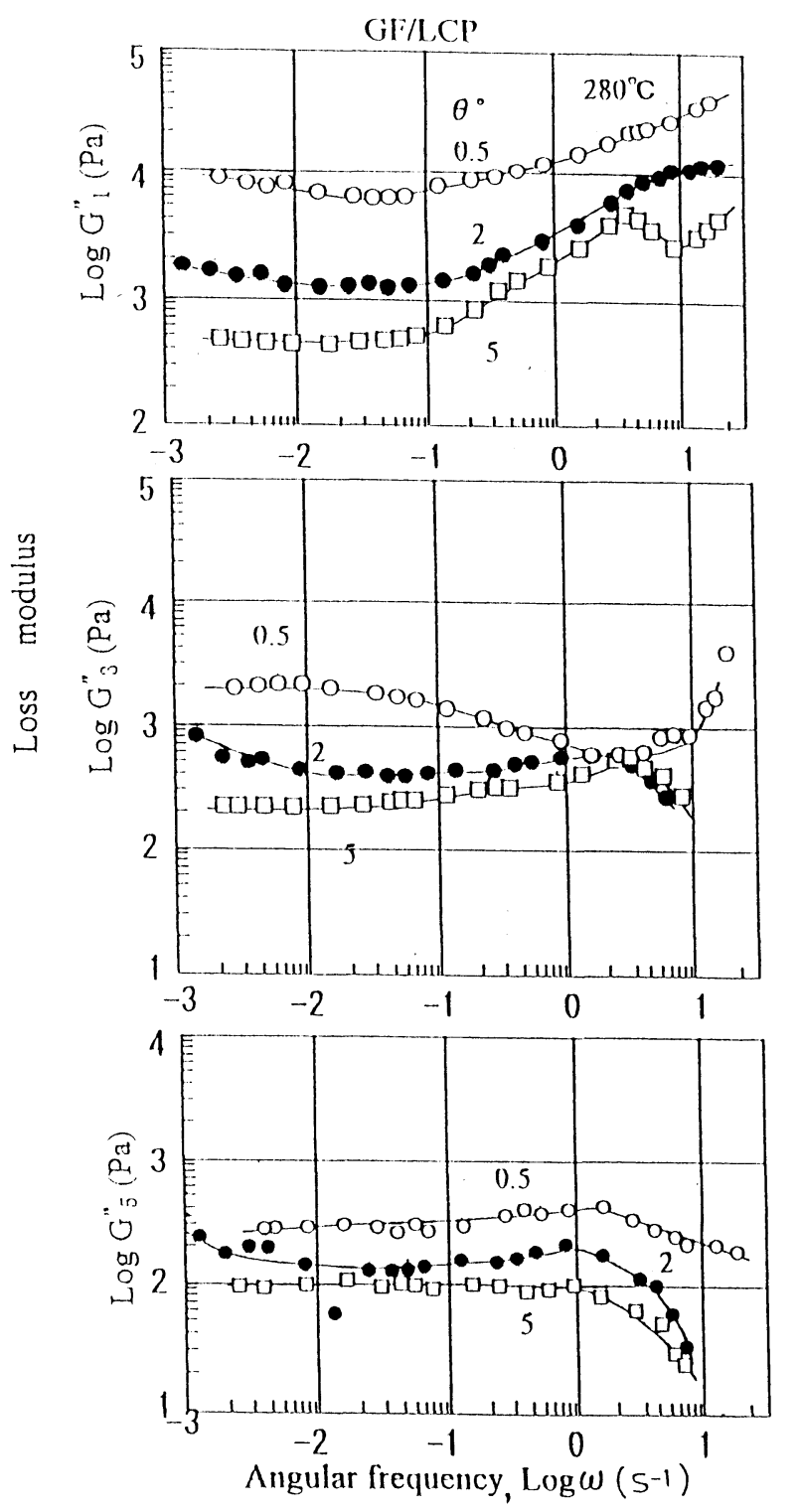

Fig.8 Loss modulus ( $\mathrm{G}{ }_{1}, \mathrm{G}{ }_{3}$ and $\mathrm{G}{ }_{5}$ ) of GF/LCP as a function of angular frequency at various oscillatory angles.

$\mathrm{G}{ }_{\mathrm{i}}$ curves of GF/LCP at $\theta=0.5^{\circ}$ show definite plateau region as in the case of $\mathrm{CF} / \mathrm{LCP}$. The relationship between $\mathrm{G}_{\mathrm{i}} / \mathrm{G}^{\prime}{ }_{\mathrm{i}}(\mathrm{i}=1,3,5)$ and $\omega$ of GF/LCP at $\theta=2^{\circ}$ as shown in Figs.7 and 8 indicates that the relation of $G{ }_{1}>\mathrm{G}{ }_{3}>\mathrm{G}{ }_{5}$ for $G{ }^{\prime}$, holds true all over the measured $\omega$ region, but that $\mathrm{G}_{1}{ }_{1} \fallingdotseq \mathrm{G}_{3}{ }_{3} \fallingdotseq \mathrm{G}_{5}{ }_{5}$ for $\mathrm{G}_{\mathrm{i}}$, in the $\omega$ region of $10^{-3}<\omega<1$ and $\mathrm{G}_{1}>\mathrm{G}_{3}>\mathrm{G}_{5}{ }_{5}$ in the high $\omega$ region. The values of the higher-order terms of GF/LCP tend to approach to those of their 1st-order terms or value as the oscillatory angle increases. This is a characteristic feature of GF/LCP, which definitely distinguishes it from CF/LCP, showing that the viscoelasticity of the former is remarkably non-linear. Figs.7 and 8 also show the relationship between $\mathrm{G}^{\prime}{ }_{\mathrm{i}}$ and $\mathrm{G}{ }_{\mathrm{i}}(\mathrm{i}=1$,
$3,5)$ and $\omega$ of GF/LCP at $\theta=5^{\circ}$. As can be seen from the figures, an increase in the oscillatory angle results in the decrease of the higher-order terms, causing a decrease in the non-linearity of the viscoelasticity of GF/LCP. For G”, the relation of $\mathrm{G}{ }_{1}>\mathrm{G}{ }_{3}>\mathrm{G}{ }_{5}$ holds true all over the measured $\omega$ region, while the three $G_{i}^{\prime}(i=1,3,5)$ curves are similar to those at $\theta=2^{\circ}$ described above, showing no significant difference in the low $\omega$ region. Specifically, the relation between the $\mathrm{G}^{\prime}{ }_{i}$ values of GF/LCP at $\theta=5^{\circ}$ in the low $\omega$ region are similar to that at $\theta=2^{\circ}$ represented as $\mathrm{G}_{1} \fallingdotseq$ $\mathrm{G}_{3}{ }_{3} \fallingdotseq \mathrm{G}_{5}$. The relation of $\mathrm{G}_{1}, \mathrm{G}_{3}$ and $\mathrm{G}_{5}{ }_{5}$ in the high $\omega$ region is given as $\mathrm{G}_{1}>\mathrm{G}^{\prime}{ }_{3}>\mathrm{G}^{\prime}{ }_{5}$. The three $\mathrm{G}^{\prime}{ }_{i}(\mathrm{i}=1,3,5)$ curves at $\theta=5^{\circ}$, the values of which are larger than those of their respective counterparts of CF/LCP as shown in Fig. 4 and others, indicate that the viscoelasticity of GF/LCP is markedly non-linear.

In order to identify the extent of the non-linearity of the viscoelastic behavior of CF/LCP and GF/LCP, two kinds of ratio $G{ }_{3} / G{ }_{1}$ and $G{ }_{3} / G{ }_{1}$ are shown as a function of $\omega$ in Fig. 9 and Fig.10, respectively.

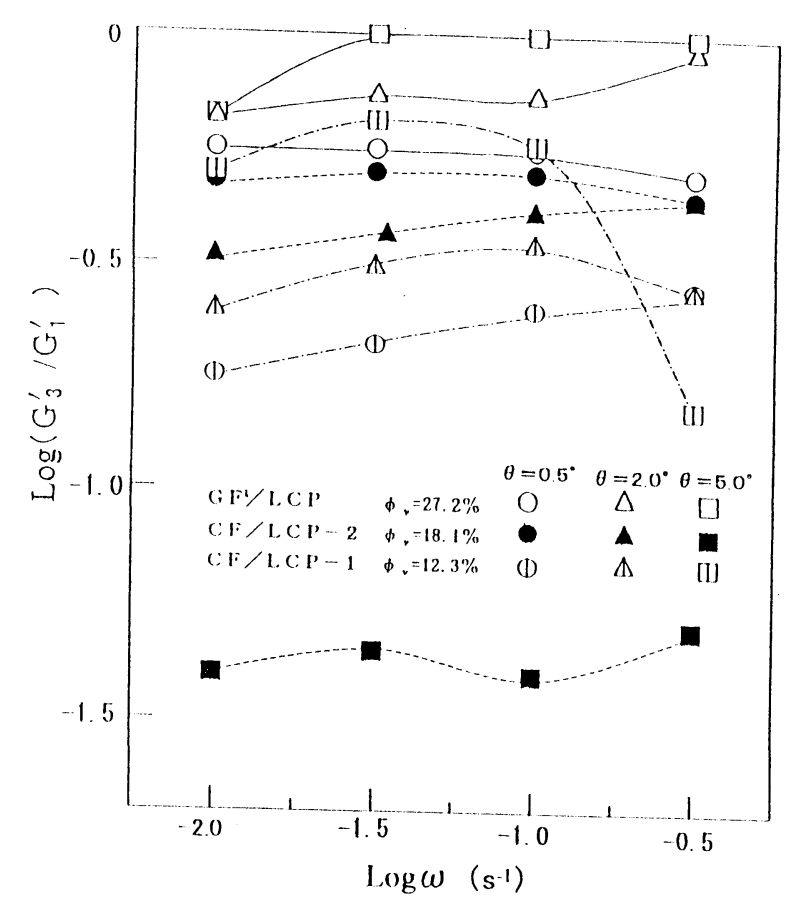

Fig.9 Storage modulus ratio ( $\mathrm{G}_{3} / \mathrm{G}_{1}{ }_{1}$ ) of CF/LCP-1, CF/LCP-2 and GF/LCP as a function of angular frequency at various oscillatory angles.

From Fig.9, both CF filled LCP (CF/LCP-1, CF/LCP-2) and GF filled LCP (GF/LCP) are found to give approximately 0.3 as their $\mathrm{G}_{3} / \mathrm{G}^{\prime}{ }_{1}$ values on the whole. It can also be noted from Fig.9 that CF/LCP-1 shows a larger dependency of $\mathrm{G}_{3} / \mathrm{G}^{\prime}{ }_{1}$ on $\theta$ than that of CF/LCP-2. From the figure, it was confirmed that the non-linearity of the 


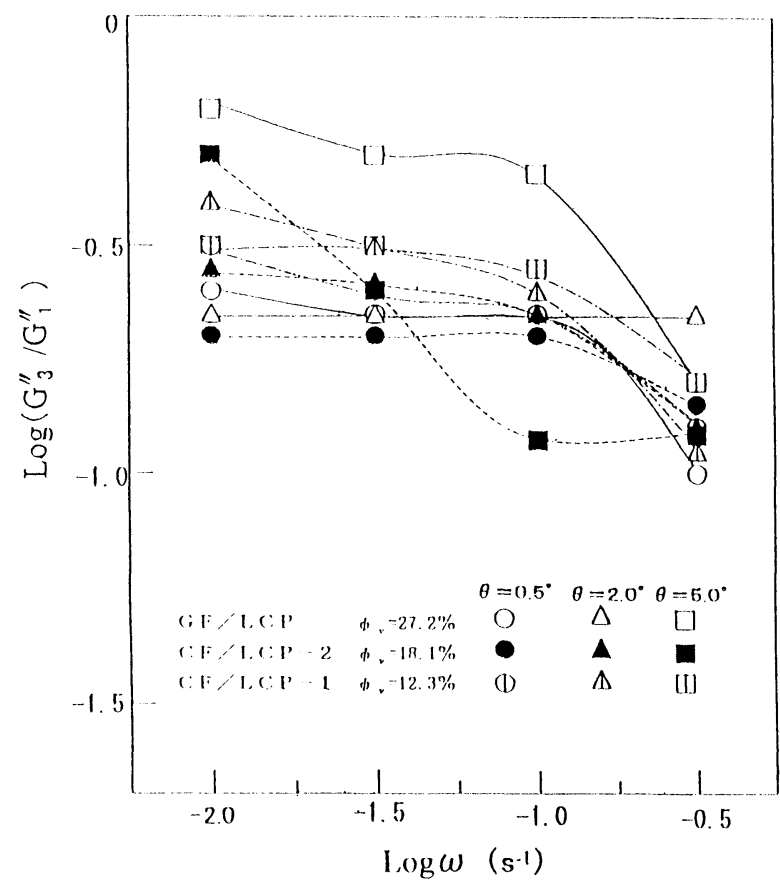

Fig.10 Loss modulus ratio ( $\mathrm{G}_{3} / \mathrm{G}_{1}$ ) of CF/LCP-1, CF/LCP-2 and GF/LCP as a function of angular frequency at various oscillatory angles.

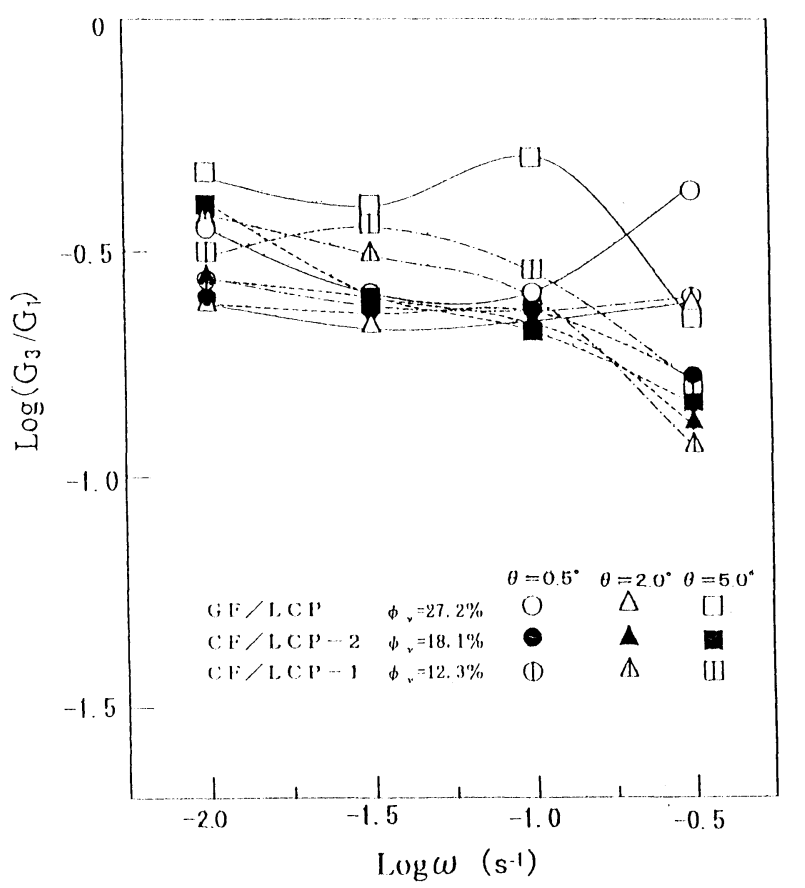

Fig. $11 \mathrm{G}_{3} / \mathrm{G}_{1}$ of CF/LCP-1, CF/LCP-2 and GF/LCP as a function of angular frequency at various oscillatory angles. viscoelastic behavior of $\mathrm{CF} / \mathrm{LCP}$ is more influenced by the length of the fiber than its fiber content.

From Fig.10 showing the relationship between $G{ }_{3} / G{ }^{\prime}$, and $\omega$ for fiber filled LCP, it can be seen that all the three specimens give small $G{ }_{3} / G{ }_{1}$ values on the whole. This result suggests that these systems show linear viscoelastic behavior. This flow behavior was more markedly noted in GF/LCP than in CF/LCP, especially in the high $\omega$ region.

The equation (5) given below was applied to fiber filled LCP in order to more clarify the non-linearity of vicoelasticity of fiber filled LCP.

$$
G_{i} / G_{1}=\left[\left(G_{i}{ }^{2}+G^{\prime}{ }_{i}^{2}\right) /\left(G_{1}{ }^{2}+G^{\prime \prime}{ }_{1}^{2}\right)\right]^{1 / 2}
$$

where the ratio, $G_{i} / G_{1}(i=3,5)$, if smaller, indicate that the viscoelastic behavior is more linear. The $G_{i} / G_{1}$ values especially for linear viscoelastic materials are equal to zero.

The calculated values of the ratio $G_{i} / G_{1}$ for $i=3$ are shown in Fig.11. The comparison of the data in Fig.11 with the $\mathrm{G}_{3} / \mathrm{G}_{1}$ and $\mathrm{G}{ }_{3} / \mathrm{G}{ }_{1}$ data in Fig.9 and Fig.10 shows that the $\mathrm{G}_{3} / \mathrm{G}_{1}$ data for two CF/LCP is considerably similar to their $\mathrm{G}_{3} / \mathrm{G}{ }_{1}$ data in Fig.10. As a summary the results obtained in the present research for $\mathrm{CF}$ and $\mathrm{GF}$ filled systems as the ratios $\mathrm{G}_{3} / \mathrm{G}_{1}{ }_{1}$ (Fig.9), G" ${ }_{3} / \mathrm{G}{ }_{1}{ }_{1}$ (Fig.10) and $\mathrm{G}_{3} / \mathrm{G}_{1}$ (Fig.11) are considered to be suitable for evaluation of the nonlinearity of their respective viscoelastic behavior. These results also show that the differences between the values of the 1 st-order terms and those of the high-order terms of $\mathrm{G}^{\prime}{ }_{i}$ and $\mathrm{G}^{\prime}{ }_{i}$ in the high $\omega$ region become smaller for GF/LCP than for CF/LCP.

\section{Conclusion}

The melts of a semi-aromatic liquid crystalline polymer (LCP) and its short-fiber composite systems filled with carbon fiber (CF) and glass fiber (GF) were measured for their dynamic viscoelastic properties to investigate the effects of the fiber type, fiber length and also strain amplitude on the non-linearity of their viscoelastic behavior experimentally by the Fourier expansion analysis. The results are as follows:

1) The steady shear flow properties of LCP melts used in this study show moderately non-Newtonian compared with those of fully aromatic liquid crystalline polymers (vectra), however both CF and GF filled LCP show remarkable non-Newtonian flow behavior.

2) The Fourier expansion analysis of unfilled LCP for storage modulus $G_{i}^{\prime}(i=1,3)$ and loss modulus $G^{\prime}{ }_{i}(i=1$, 3) shows that the values of their respective 3rd-order terms $\mathrm{G}_{3}$ and $\mathrm{G}_{3}$ are negligibly small compared with those of their respective 1st-order terms $G^{\prime}{ }_{1}$ and $G^{\prime}{ }_{1}$, suggesting that its viscoelastic flow behavior is nearly linear. In addition, the unfilled LCP shows small dependency of $\mathrm{G}^{\prime}{ }_{i}$ and $\mathrm{G}{ }_{i}$ on the strain amplitude. 
3) Two types of CF filled LCP - CF/LCP-1 and CF/LCP-2 - were subjected to similar analysis to investigate the dependency of their $G_{i}^{\prime}(i=1,3,5)$ and $G^{\prime}{ }_{i}(i=1,3,5)$ values on angular frequency, $\omega$ and oscillatory angle $\theta$ (or strain amplitude $\gamma$ ). Both CF/LCP specimens show a decrease in the values of $\mathrm{G}_{3}, \mathrm{G}{ }_{3}, \mathrm{G}{ }_{5}$ and $\mathrm{G}{ }_{5}$ from those of $\mathrm{G}^{\prime}{ }_{1}$ and $G{ }^{\prime}{ }_{1}$ in sequence with a rise in the oscillatory angle $\theta$ (strain amplitude $\gamma$ ), which means the dependency of $\mathrm{G}_{{ }_{\mathrm{i}}}$ and $\mathrm{G}{ }_{\mathrm{i}}$ on $\theta(\gamma)$.

4) GF filled LCP specimen was similarly analyzed to give $\log G_{i}\left(G^{\prime}{ }_{i}\right)-\log \omega$ curves, which showed definite plateau regions similarly to those of CF/LCP. Although compared to the latter, the ratios of the higher-order $\mathrm{G}^{\prime}{ }_{i}$ and $\mathrm{G}{ }{ }_{i}$ (especially $\mathrm{G}{ }_{3}$ and $\mathrm{G}{ }_{3}$ ) to their 1 st-order counterparts are large. This suggests that the viscoelastic behavior of GF/LCP is more non-linear than that of CF/LCP. In addition, the higher-order term values showed a tendency to increase with a rise in the strain amplitude, approaching the 1 st-term values. Compared to CF/LCP, GF/LCP showed a small difference between the values of the 1st-order terms of $\mathrm{G}_{\mathrm{i}}{ }_{\mathrm{i}}$ and $\mathrm{G}{ }^{\prime}{ }_{i}$ and those of their respective high-order counterparts in the high $\omega$ region.

5) The comparison of the three CF or GF filled LCP showed that there was no significant difference between them in terms of the dependency of their respective ratios, $\mathrm{G}{ }_{3} / \mathrm{G}{ }_{1}, \mathrm{G}{ }_{3} / \mathrm{G}{ }_{1}$ and $\mathrm{G}_{3} / \mathrm{G}_{1}$, which represent the extent of the non-linearity of their viscoelastic behavior, on $\omega$, although the ratio $G{ }_{3} / G "{ }_{1}$ of GF/LCP were found to be larger than those of CF/LCP. This reflects marked nonlinearity in the loss modulus of GF/LCP, especially in the high $\omega$ region.

\section{References}

[1] Araki, K., Kitano, T. Uyama, A. Unryu, T.; Seikei Kakou, 10, 357(1998)

[2] Araki, K., Kitano, T. Uyama, A. Unryu, T.; Seikei Kakou, 10, 539(1998)

[3] Araki, K., Nakamura, K.; J. Text. Mach. Soc. Japan, 41, 47(1988)

[4] Araki, K., Nakamura, K.; J. Text. Mach. Soc. Japan, 42, 99(1989)

[5] Araki, K., Nakamura, K.; J. Text. Mach. Soc. Japan, 42, T135(1989)

[6] Araki, K., Kitano, T. Uyama, A. Unryu, T.; Seikei Kakou, 12, 52(2000)

[7] Araki, K., Kitano, T. Uyama, A. Unryu, T.; Seikei Kakou, 12, 582(2000)

[8] Araki, K., Kitano, T., Maegawa, Z.; J Applied. Rheolgy, submitted.

[9] Araki, K., Kitano, T., Maegawa, Z.; Seikei Kakou, submitted.

[10] Kitano, T., Kataoka, T.; Rheol, Acta, 19, 753(1980)

[11] Sakai, S., Kitano, T., Nishimura, T.; Kobunshi Ronbunshu., 45, 123(1988)

[12] Chung, T, S.; Polym. Eng, Sci, 26, 901(1986)

[13] Matsumoto, T., Segawa, Y., Warashina, Y., Onogi,S.; Trans, Soc, Rheol, 17, 47(1973)

[14] Watanabe, H., Kodaka, T.; Nihon Reoroj Gakkaishi, 8, 26(1980)

[15] Onogi, S., Matsumoto, T.; Polym. Eng. Rev. 1, 45 (1981)

[16] Watanabe, H., Kataoka, T., Shibayama, M., Kawai, H.; J. Rheol. 26, 153(1982)

[17] Cheremisinoff, N, P., Cheremisinoff, P, N.; "Handbook of Applied Polymer Processing Technology", Marcel Dekker, Inc, New York(1996) 